This item was submitted to Loughborough's Research Repository by the author.

Items in Figshare are protected by copyright, with all rights reserved, unless otherwise indicated.

\title{
Droplet factories: Synthesis and assembly of metal nanoparticles on magnetic supports
}

\section{PLEASE CITE THE PUBLISHED VERSION}

https://doi.org/10.1016/j.jcis.2020.02.087

\section{PUBLISHER}

Elsevier BV

VERSION

AM (Accepted Manuscript)

\section{PUBLISHER STATEMENT}

This paper was accepted for publication in the journal Journal of Colloid and Interface Science and the definitive published version is available at https://doi.org/10.1016/j.jcis.2020.02.087.

\section{LICENCE}

CC BY-NC-ND 4.0

\section{REPOSITORY RECORD}

Sachdev, Suchanuch, Rhushabh Maugi, Sam Davis, Scott Doak, Zhaoxia Zhou, and Mark Platt. 2020. "Droplet Factories: Synthesis and Assembly of Metal Nanoparticles on Magnetic Supports". figshare.

https://hdl.handle.net/2134/11897406.v1. 


\title{
Droplet factories: Synthesis and Assembly of Metal Nanoparticles on
} Magnetic Supports.

\author{
Suchanuch Sachdev ${ }^{1}$, Rhushabh Maugi ${ }^{1}$, Samuel Davis ${ }^{2}$, Scott S Doak ${ }^{2}$, Zhaoxia Zhou ${ }^{2}$, \\ Mark Platt ${ }^{1 *}$
}

1 - Department of Chemistry, Loughborough University, Loughborough, LE11 3TU, United Kingdom.

2 - Loughborough Materials Characterisation Centre, Department of Materials, Loughborough University, Loughborough, LE11 3TU, United Kingdom

* Email: m.platt@lboro.ac.uk; Tel: +441509 222573 


\begin{abstract}
The interface between two immiscible liquids represent an ideal substrate for the assembly of nanomaterials. The defect free surface provides a reproducible support for creating densely packed ordered materials. Here a droplet flow reactor is presented for the synthesis and/ or assembly of nanomaterials at the interface of the emulsion. Each droplet acts as a microreactor for a reaction between decamethylferrocene $(\mathrm{DmFc})$ within the hexane and metal salts $\left(\mathrm{Ag}^{+} /\right.$ $\left.\mathrm{Pd}^{2+}\right)$ in the aqueous phase. The hypothesis was that a spontaneous, interfacial reaction would lead to the assembly of nanomaterials creating a Pickering emulsion. The subsequent removal of the solvents showed how the Ag nanoparticles remain trapped at the interface and retain the shape of the droplet, however the Pd nanoparticles were dispersed with no tertiary structure. To further exploit this, a one-step process where the particles are synthesised and then assembled into core-shell materials was proposed. The same reactions were performed in the presence of oleic acid stabilised iron oxide nanoparticles dispersed within the hexane. It was shown that by changing the reaction rate and ratio between metal and iron oxide a continuous coating of metal nanoparticles can be formed on top of an iron oxide microsphere, or form a uniform composite. These insights offer a new method and chemistry within flow reactors for the creation of palladium and silver nanoparticles. We use the technique to create metal coated iron oxide nanomaterials but the methodology could be easily transferred to the assembly of other materials.
\end{abstract}

Key words: Microfluidic, Emulsion, Liquid-Liquid, Pickering Emulsion, Core-shell 


\section{Introduction}

The size, shape, crystal structure and surface properties can all affect the properties of metallic nanoparticles. Anisotropic particles can further expand the number of applications producing enhanced properties for example for the treatment of tumours ${ }^{1}$, enhanced Raman detection ${ }^{2}$ and optical sensors ${ }^{3}$. A further enhancement of properties can be done via the creation of dual functionality materials in the form of Janus, or Core-shell particles. Materials that contain magnetic properties are particularly desirable as they respond to an externally applied magnetic field, allowing them to be manoeuvred in three-dimensional space with a handheld magnet ${ }^{4}$. Such particles have applications within targeted drug delivery, the rapid purification of biological products and catalysis.

The traditional route for synthesising materials is to mix the chemicals within a flask or beaker, and after sufficient time has passed for the reaction to complete, extraction and analysis of the products. These workflows are often referred to as batch reactors. ${ }^{5}$ The translation of batch chemistries onto continuous flow platforms represent an area of increased research over the last decade. They offer lower production costs, as well as reducing the variation in products from batch-to-batch. These "lab-on-a-chip" devices guarantee uniform mixing, and fluidic behaviour with integrated continuous inline monitoring of chemical products for high-throughput processing. An important factor in the controlled synthesis and assembly of materials is the choice of surfactant and capping ligand. A potential route to use ligand free assembly is to use the interface between two liquids. The interface between two immiscible liquids offers a defect free, reproducible substrate to grow metals. ${ }^{6-12}$ This allows the material to be easily recovered, as the majority of the formed particles remain at the interface upon creation ${ }^{13}$. The interface between two liquids can also be used to assemble nanoparticles with their spacing and composition at the interface with further control with the use of ligands ${ }^{14}$. The assembly of nanomaterials at the liquid-liquid (liq-liq) interface is spontaneous due to the favourable stabilisation of the interfacial free energy and was first discovered by Ramsden ${ }^{15}$ and Pickering ${ }^{16}$. The preferred location at the interface is determined by the Young's equation and can be controlled by tailoring the surface chemistry of the particles. The contact angle of the particle at the interface can be controlled and can be used to create oil-in-water or water-in-oil emulsions, known generally as Pickering Emulsions. Pickering emulsion synthesis strategies have assembled a range of both polymer and inorganic materials at the interface of the droplets ${ }^{17-20}$, although predicting the behaviour of nonspherical particles at such interfaces is challenging but simulations can offer insights into mechanisms.

In this paper we present a strategy for producing $\mathrm{Ag}$ and $\mathrm{Pd}$ nanoparticles at the interface of hexane/water emulsions, figure 1a. Using a spontaneous interfacial reaction between decamethylferrocence $(\mathrm{DmFc})$, in hexane and metal salts in the aqueous phase that was previously reported by our group for the synthesis of gold, Au, nanoparticles, NP's. ${ }^{21,22}$ The materials are formed via an interfacial reaction, figure 1ai, however their stability at the interface is material dependent, figure 1aii. The hypothesis was that the removal of the organic phase would allow particles trapped at the droplet interface to assemble into clusters, figure 1aii. We observed that in the absence of any stabilising ligands the Ag particles maintain the shape of the droplets forming clusters of micron sized aggregates. Whereas the $\mathrm{Pd}$ nanoparticles disperse through the liquid and do not hold any tertiary structure when imaged via the electron microscope, figure 1aiii. Upon the addition of iron oxide nanoparticles, figure 
$1 \mathrm{~b}$ and bi, to the hexane phase, the same reactions should form a metal shell around a hexane core of iron oxide, figure 1bii, figure 1c. It was hypothesised that removing the hexane would allow the metal shell to assemble around the magnetic core, and that the quality of the shell would be dependent upon rate of reaction and nanoparticle formation. In the case of the faster forming Ag particles the presence of the magnetic core decreases the stability of the particles at the interface and do not form a coherent core-shell material. Whereas the slower Pd grown particles are stabilised and coated onto the iron oxide core. The ratio of Pd to iron oxide is key in forming a stable and continuous core-shell structure. Whilst the reaction rate is shown to be faster for silver, leading to the hypothesis that a nanoparticle shell should form quickly around the core, other factors such as nanoparticle size and nucleation rate all play a role. We use the technique to create metal coated iron oxide nanomaterials but the methodology could be easily transferred to the assembly of other materials.

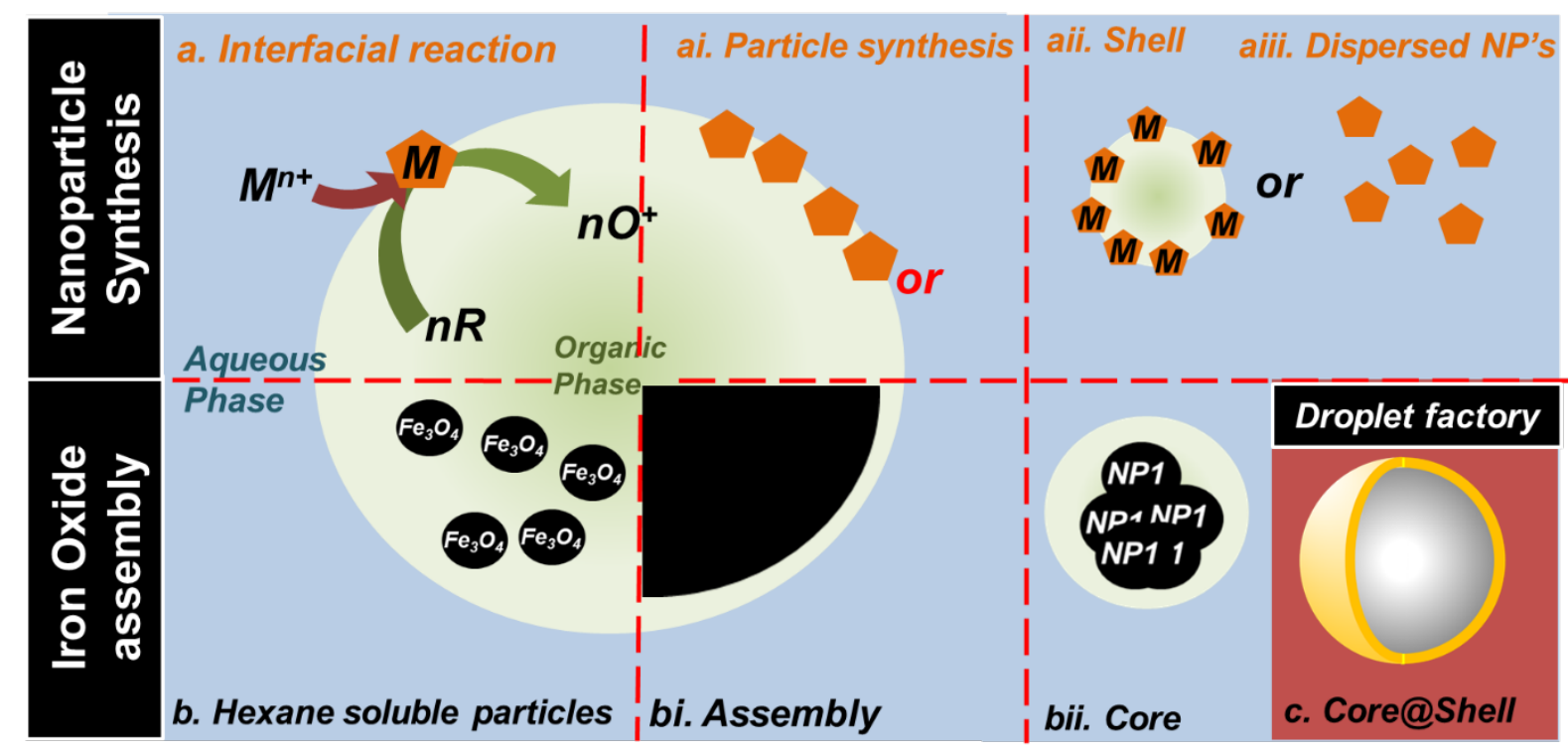

Figure 1 schematic of the reaction and growth mechanism.

\section{Methods and materials}

\section{Chemicals and reagents}

The following chemicals were purchased from Sigma-Aldrich, U.K., and unless stated otherwise the chemicals were used without purification. Sodium dodecyl sulfate (SDS) (436143), iron(II) chloride tetrahydrate $\left(\mathrm{FeCl}_{2} \cdot 4 \mathrm{H}_{2} \mathrm{O}\right)$ (220299), iron(III) chloride hexahydrate $\left(\mathrm{FeCl}_{3} \cdot 6 \mathrm{H}_{2} \mathrm{O}\right)$ (236489), ammonium hydroxide $\left(\mathrm{NH}_{3} \cdot \mathrm{H}_{2} \mathrm{O}\right)$ (338818), oleic acid (364525), perchloric acid (244252), sodium tetrachloropalladate (205818), sodium tetrachloroplatinate (432857), bis(pentamethylcyclopentadienyl) iron(II) (97\%, 378542), silver nitrate (99\%, 204390) and Potassium Hexcyanoferrate. Deionized (DI) water with a resistivity of 18.2 $\mathrm{M} \Omega \cdot \mathrm{cm}$ was used throughout. Synthesis of hydrophobic nanomagnetic particles $(250 \mathrm{~mL})$ scale. $\mathrm{FeCl}_{12} \cdot 4 \mathrm{H}_{2} \mathrm{O}(12 \mathrm{~g})$ and $\mathrm{FeCl}_{3} \cdot 6 \mathrm{H}_{2} \mathrm{O}(24.5 \mathrm{~g})$ were dissolved in DI-water $(62.5 \mathrm{~mL})$ in a 250 $\mathrm{mL}$ three neck bottle. The flask was placed in an ice bath. $\mathrm{NH}_{3} \cdot \mathrm{H}_{2} \mathrm{O}(50 \mathrm{~mL})$ was added rapidly with vigorous stirring. The flask was left in the ice bath for 45 minutes. The solution was rapidly heated to $85^{\circ} \mathrm{C}$ for 1 hour. Oleic acid $(7.5 \mathrm{~mL})$ was then added and the solution was further heated for another 1 hour. The flask was cooled to room temperature and the slurry was 
transferred to a $150 \mathrm{~mL}$ beaker. Slurry was washed 3 times with ethanol $(50 \mathrm{~mL})$ and each time the black magnetite was collected using a block magnet. The slur was then washed 3 times with DI-water $(50 \mathrm{~mL})$ before being washed 3 times with $20 \%$ perchloric acid $(50 \mathrm{~mL})$ to dissolve $\mathrm{Fe}(\mathrm{OH})_{2}$ and $\mathrm{Fe}(\mathrm{OH})_{3}$. The slur was again washed 3 times with DI-water $(50 \mathrm{~mL})$, before being washed three times with ethanol $(50 \mathrm{~mL})$. Hexane $(87.5 \mathrm{~mL})$ was added to the resultant particles so to disperse well, this was called ferrofluid. The particle concentration was determined by weighing the mass of material that remained after the evaporation of a known volume of solvent.

Microfluidics.

Microfluidics instruments were supplied by Dolomite, a $14 \mu \mathrm{m}$ etch depth Dolomite hydrophilic x-junction "small droplet chip" (Part No. 3200136), Dolomite Mitos P-Pump basic (Part No. 3200175) were controlled via the Dolomite Mitos Flow Control Centre Version 2.2.15. Detailed information on the experimental section can be found elsewhere. ${ }^{22}$ Briefly the chip is made from a hydrophilic silica, the narrow channel where the organic phase and aqueous phase from the droplets is $14 \mu \mathrm{m}$ in diameter, which expands to a $500 \mu \mathrm{m}$ channel before exiting into the collection tube. The formation of the nanoparticles uses the method described in detailed elsewhere. ${ }^{22}$

Free standing liquid-liquid reaction and kinetics.

A free standing interface was created in a glass vial. For each measurement a fresh interface was prepared under the same chemical conditions as found in the droplet reactors i.e. using a hexane/SDS/water interface. The absorbance of the hexane solution was measured at $0,2,5$ and 10 min intervals at a wavelength of $424 \mathrm{~nm}$.

\section{Collection of particles.}

The nanomaterials were centrifuged (12000 rpm, 5 mins) then the supernantant was discarded and replaced with acetonitrile $(500 \mu \mathrm{L})$. The washing process was repeated with DI-water. The sample was then re-dispersed in DI-water before imaging. When the sample contained iron oxide nanoparticles, the solution is purified by placing on a magrack (GE, healthcare, UK) for 2 min. The supernatant was discarded and replaced with DI-water $(100 \mu \mathrm{L})$ then it was placed on a sonication bath for $1 \mathrm{~min}$. The washing process was repeated three times with DI-water and twice with acetonitrile. The sample was then re-dispersed in either water or acetone (100 $\mu \mathrm{L})$.

\section{Imaging}

Scanning electron microscopy (SEM) samples were prepared by drop-casting the suspension onto conductive copper tape. The SEMs used for imaging were a table top SEM Hitachi TM3030, two FEG-SEMs JEOL 7800F and Leo (Zeiss) 1530-VP. Transmission electron microscopy (TEM) specimens were prepared via two routes. (i) For nanoparticles size and crystallinity, suspensions were ultrasonicated and then drop-cast onto standard holey carbon supported TEM grids. (ii) For cross-sections of the core shell structures, the ultrasonicated suspensions were drop-cast onto a plasma treated Si wafer for optimised particle dispersion on the Si surface, hence single particle cross-section. An FEI Nova 600 Nanolab dual beam (focused ion beam (FIB) and SEM) equipped with EDS was employed to extract cross sections 
from the core-shell particles (see supplement). TEMs equipped with Oxford Instruments energy dispersive X-ray spectroscopy (EDS) system were used to characterise the samples, a Jeol 2000FX and a FEI Tecnai F20. Both were operated at 200kV accelerating voltage. Selected area electron diffraction (SAED) patterns were recorded to identify the crystallinity of the particles. Elemental maps were acquired to track the distribution of the nanoparticles.

\section{Results and discussion.}

\section{Nanoparticle synthesis using droplets.}

Palladium and silver were chosen to continue the development of core-shell droplet reactors as they have been reported to form spontaneous reactions with ferrocence derivatives at the liqliq interface. ${ }^{23-25}$ There are two main driving forces during the metal deposition. The first is the lowering of the free energy of the interface i.e. the sum of the organic/NP and water/NP are lower than the organic/water interaction. The second is the auto catalytic nature of the reaction once a metal nanoparticle is formed. During nucleation the reactants must come together to nucleate the material, once a metal particle is formed electrons can be shuttled along through the particles to react with ions anywhere along their surface. The nucleation rate and growth kinetics are not always related to the redox potential of the reactants. Before the reactions were performed in a droplet, they were first carried out on a large planar interface, the reaction rate was followed by monitoring the colour change as $\mathrm{DmFc}$ is oxidised. Figure 2 shows the changes in DmFc concentration as a function of time for different metal ions, as a control the hexacyanoferrate ion was also added to one experiment. The standard redox potentials would suggest that the rate of the reaction should be $\mathrm{Au}>\mathrm{Ag}>\mathrm{Pd}>\mathrm{Fe}\left(\mathrm{CN}_{6}\right)^{3-}$, however the measured rates were $\mathrm{Ag}>>\mathrm{Fe}\left(\mathrm{CN}_{6}\right)^{3-}>\mathrm{Pd}>\mathrm{Au}$. The deviation from this series is caused by differences in the reaction kinetics although the reasons for the deviation from the expected trend, based upon the reduction potentials, is not clear at present.

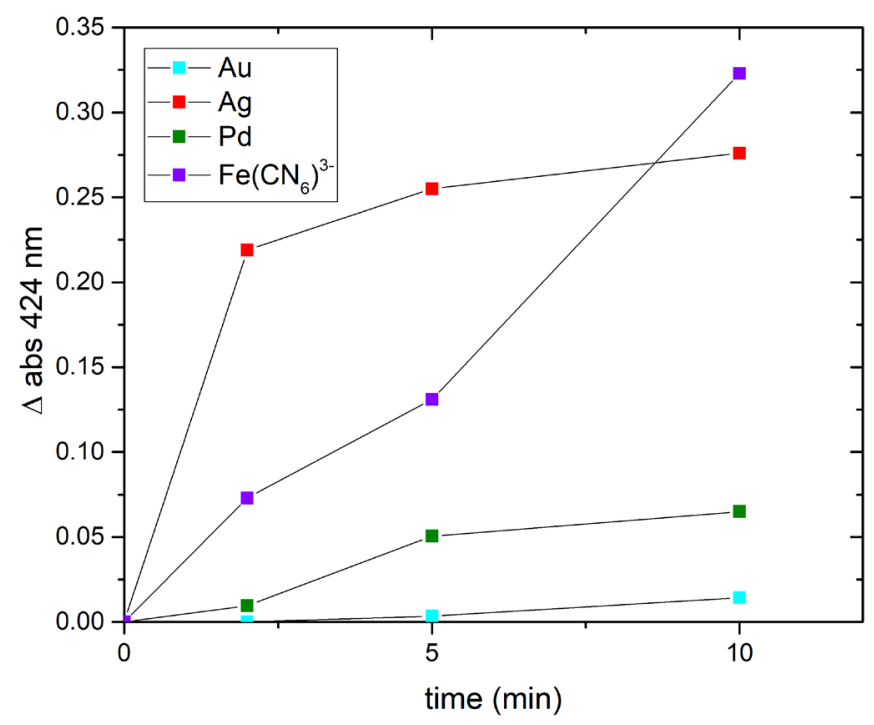

Figure 2 Change in absorbance at $424 \mathrm{~nm}$ versus time. The volume of hexane and DI water was $3 \mathrm{~mL}$. The initial concentration of the metal ions were $5 \mathrm{mM}$ and the DmFc $5 \mathrm{mM}$. 
Figure 2 shows rate of formation of $\mathrm{DmFc}^{+}$versus time, at planar liquid-liquid interfaces. The reaction was carried out at a similar interface to the droplet i.e. hexane/SDS/Water. For the reaction with $\mathrm{Ag}$, there was a rapid change in DmFc concentration, after 2 mins no change in absorbance was observed. In contrast the same reaction with $\mathrm{Au}$ took 5 mins before a significant change was measured in the UV-Vis. This work aimed to form a complete metal NP layer around each droplet. Thus the faster Ag reaction looked promising as it might form the shell quickly.

The next stage was to transfer the reaction into the droplets. Emulsion droplets were created within a microfluidic chip via a flow focusing strategy reported elsewhere. ${ }^{22}$ The droplet chip had a $14 \mu \mathrm{m}$ restriction at its narrowest point. The size of the droplets was controlled by the chip dimensions as well as the flow rate of the hexane and aqueous phase. As the chips dimensions remain constant throughout the experiment, the droplet size is varied by the relative flow rates of the liquids. As the newly formed droplets required a surfactant to stabilise them, SDS was present in the experiments simply to provide stability to the emulsions prior to the formation of metal NP's. The droplets were first made in the presence of SDS, with each droplet containing the DmFc for the reaction. The droplets were then flowed through a solution containing the metal ion of choice (Ag or Pd). ${ }^{22}$ The droplets were mixed with the metal ion solution for a period of 1 hour, after which the stir bar was removed and the hexane allowed to evaporate (30 mins). After which the precipitate at the bottom of the beaker was prepared for imaging as described above. The products of the Ag reaction are shown in figure 3a-c. The SEM image in figure 3a shows spherical clusters of NP's, circa $800 \mathrm{~nm}$ in size, there are also some smaller aggregates of NP's present on the imaging stub. TEM images of some of the clusters show NP's with an average size of $12 \pm 2 \mathrm{~nm}$ in size. The diffraction patterns confirmed a nanocrystalline material, and it's interesting to note that some of the AgNP's were nanosheets, figure $3 \mathrm{~b}$ insert (arrowed), and similar to the Au particles formed previously. ${ }^{22}$ These were large single crystal Ag sheets, as indicated by the discrete spots of strong intensity coinciding on the rings of the nanocrystalline Ag diffraction pattern as shown in figure $3 \mathrm{c}$. The discrete diffraction spots, of the Ag (Figure3c) also suggests likely individual Ag particles of large size in the order $\sim 100 \mathrm{~nm}$. For the Pd reaction, figures 3d-f, no larger aggregates or consistent tertiary structure was observed. The average size of the Pd NPs via TEM was $4.8 \pm$ $1.2 \mathrm{~nm}$, and the more continuous and diffused nature of the rings of the diffraction patterns of $\mathrm{Pd}$ (e.g.Fig.3f) indicates in general smaller crystal size of Pd than Ag particles as in Figure 3c. Unlike the reaction previously reported with $\mathrm{Au}$, there was no change in particle size and shape for either the Pd or Ag as the droplet diameter, and reactant ratio was varied, data not shown. 

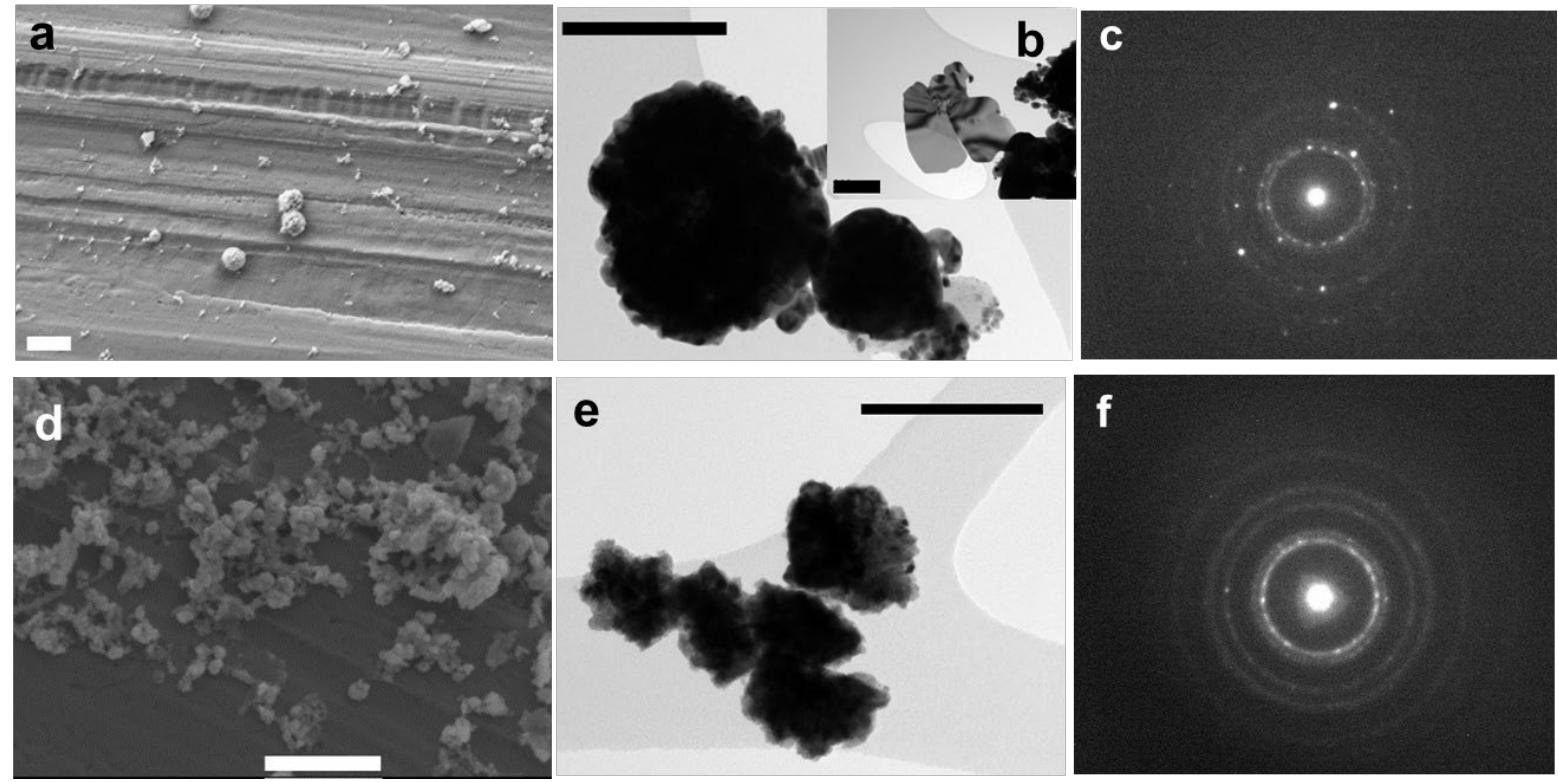

Figure 3 a-c Silver Particles. All formed under the conditions Dmfc $5 \mathrm{mM}, \mathrm{AgNO}_{3} 10 \mathrm{mM}$, droplet size 10.5 um. a, SEM, $b$ TEM with insert of Ag sheets, and c diffraction patterns of the sample. d-f Palladium Particles. All formed under the conditions Dmfc $5 \mathrm{mM}, K_{2} P d C l_{4} 5 \mathrm{mM}$, droplet size $9 \mathrm{um}$. d, SEM, e, TEM and $f$ diffraction pattern of the sample. Scale bar $1 \mathrm{um}$ in a,d, and $200 \mathrm{~nm}$ in b,e.

\section{Formation of core shell particles}

An application of this reaction was to coat/ assemble the freshly formed NP onto a core. We chose $\mathrm{Fe}_{3} \mathrm{O}_{4}$ as a core due to its inherent magnetic properties. Such particles have applications within targeted drug delivery, the rapid purification of products and catalysis. Thus the next experiment was to ascertain if core shell particles could be created, i.e. figure 1c. $\mathrm{Fe}_{3} \mathrm{O}_{4}$ nanoparticles were added to the hexane droplets along with the $\mathrm{DmFc}$, every other aspect of the setup remained the same. The $\mathrm{Fe}_{3} \mathrm{O}_{4}$ does not actively participate in the redox reaction, however it may change the viscosity of the hexanes and thus the diffusion of the DmFc. The resultant particles obtained with the Ag reaction are shown in Figure 4. 


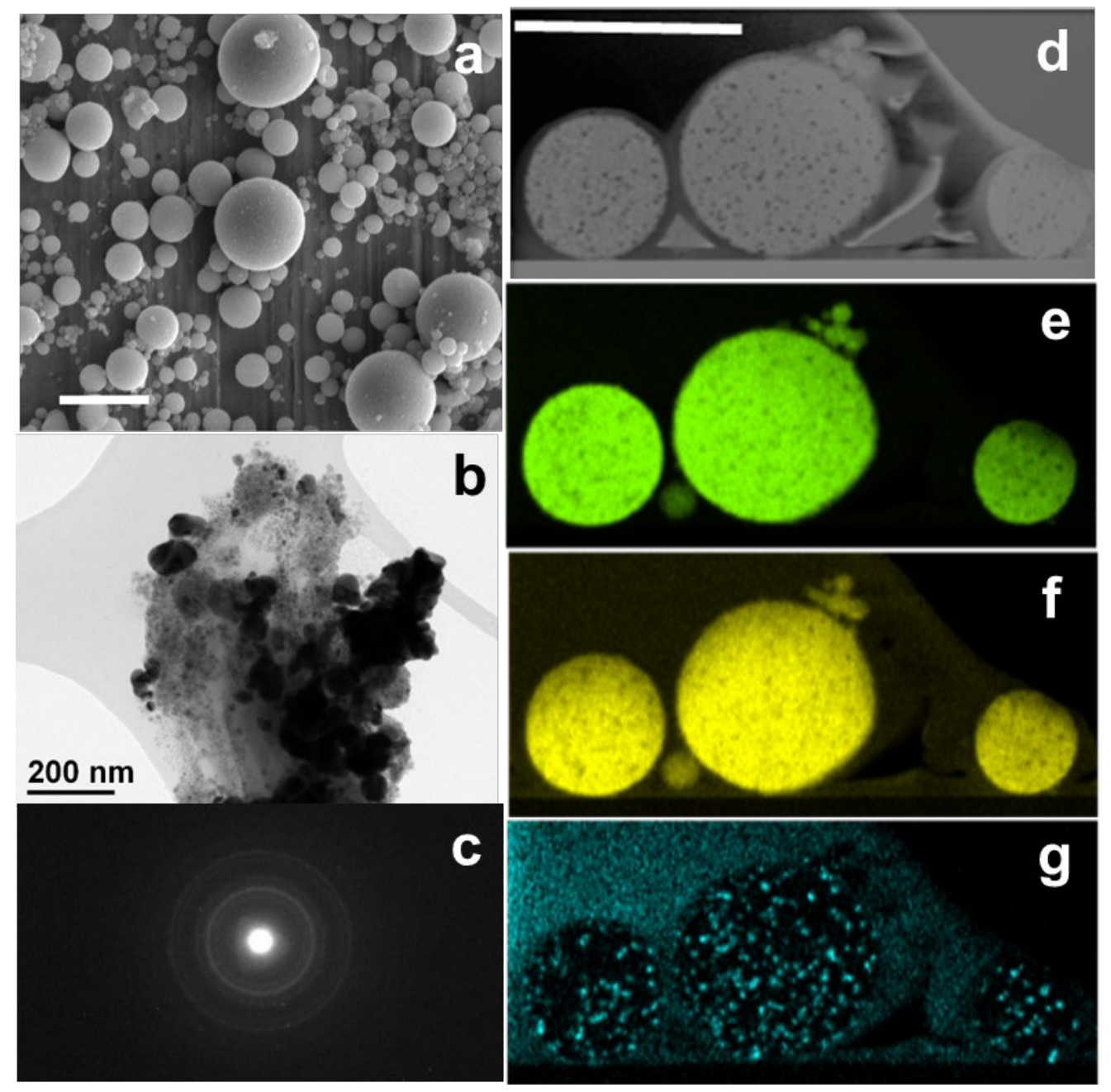

Figure 4.Ag@Fe $\mathrm{O}_{4}$ particle synthesis. $\mathrm{Fe}_{3} \mathrm{O}_{4}$ at $3.5 \mathrm{mg} / \mathrm{ml}, \mathrm{DmFc}$ at $5 \mathrm{mM}, \mathrm{AgNO}_{3}$ at $10 \mathrm{mM}$ and droplet diameter9um. a) SEM of the Ag@Fe $\mathrm{F}_{3} \mathrm{O}_{4}$ particles, scale bar $2 \mu \mathrm{m}$. b) TEM of the Ag particles within the supernatant, scale bar $250 \mathrm{~nm}$. c) SAD pattern of the particles within b. d) FIB cross section of particles with elemental mapping showing in $\mathrm{Fe}, \mathrm{O}$ and $\mathrm{Ag}$ respectively e-g. Droplet size was 10.5 $\mu m$

Figure 4a, shows the spherical particles formed in the presence of $\mathrm{Ag}$ and $\mathrm{Fe}_{3} \mathrm{O}_{4}$. Their shape is generally similar to those typically observed when only $\mathrm{Fe}_{3} \mathrm{O}_{4}$ is present, figure s1. However it was interesting to note the polydispersity of the aggregate sizes. Typically, particles made in the droplet chip are highly monodisperse, figure s1. The presence of the silver reaction, and the particles at the interface may destabilise the droplets forcing them to coalesce, which gives rise to this variance. AgNP's were also found within the supernatant, figure $4 \mathrm{~b}$, and SAD pattern in figure $4 \mathrm{c}$ suggests a much smaller crystal size then those made in figure 3 . The presence of AgNP within the solution was a little disappointing and seems to indicate that the presence of the iron oxide within the hexane, changes the crystallinity of the silver, and destabilises it at the interface. Although it cannot be determined here if the size of the NP or the presence of the $\mathrm{Fe}_{3} \mathrm{O}_{4}$ is the reason for that they are not strongly bound to the interface, and further work is needed to determine the mechanism. To check if a Ag shell had formed around the iron oxide core, a cross-section of $\sim 100 \mathrm{~nm}$ thickness was extracted from some random particles using a 
focused ion beam (FIB) and examined using a TEM. The images and subsequent elemental analysis are shown in figure $4 \mathrm{~d}$-g. The analysis of the particles shown in figure 4 reveal a solid particle, elemental analysis reveals that some Ag particles are present in each microparticles, but rather than being trapped at the surface/ exterior they appear to be dispersed throughout the entirety of the material. The mechanism by which they are internalised is unclear, as the NP's should remain trapped at the interface. During the evaporation of the solvent, the convection within the droplet may drag some NP's into the hexane, or they aggregate with the $\mathrm{Fe}_{3} \mathrm{O}_{4}$ particles which aids their uptake into the hexane, the mechanism is unclear. The experiment was repeated using the Pd salt, and the results are shown in figure 5.
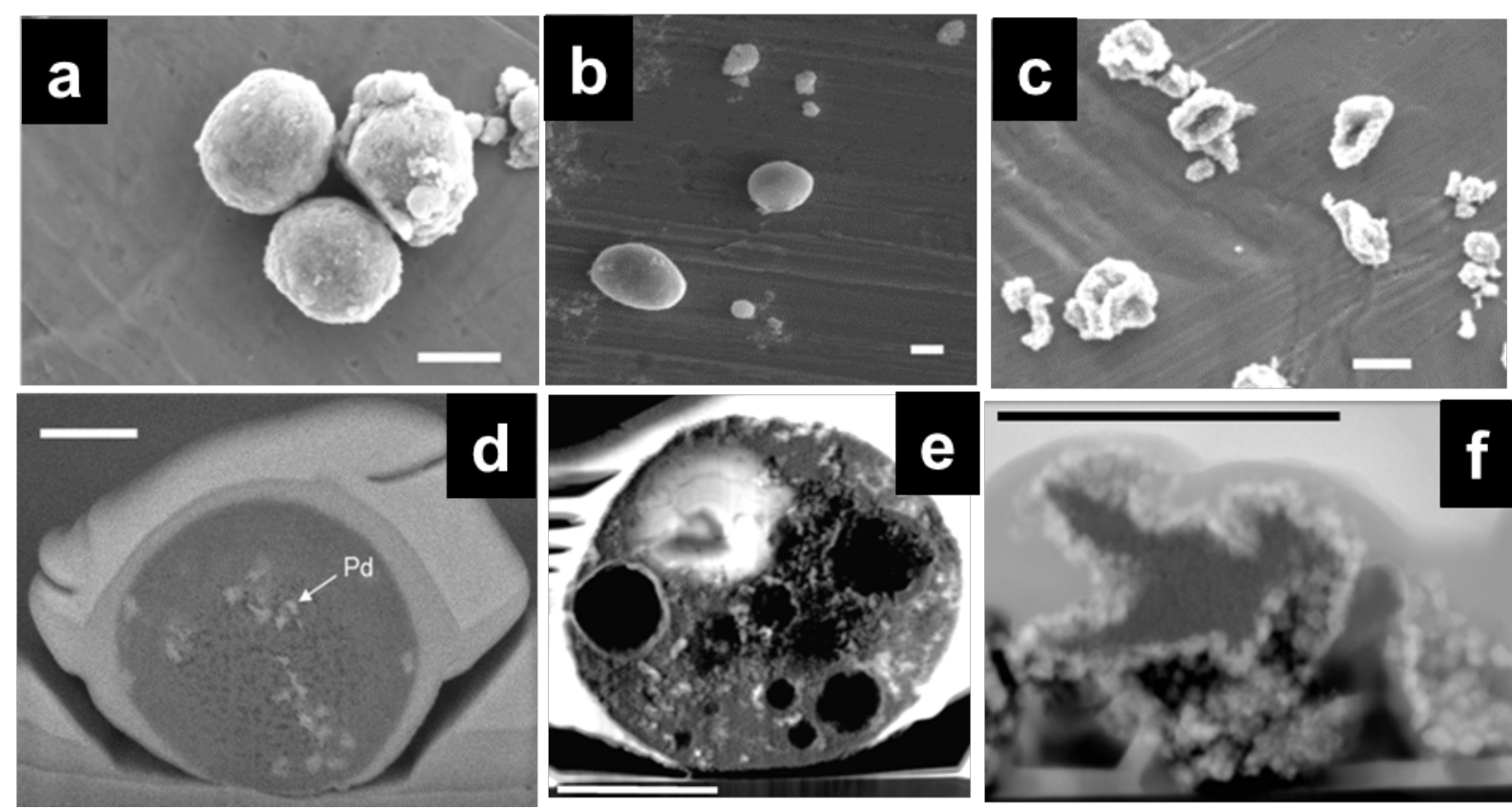

Figure 5.Pd@Fe $\mathrm{O}_{4}$ particle synthesis. For all particles the DmFc and $\mathrm{K}_{2} \mathrm{PdCl}_{4}$ was $5 \mathrm{mM}$. The concentration of $\mathrm{Fe}_{3} \mathrm{O}_{4}$ was varied from $3.5,1$ and $0.3 \mathrm{mg} / \mathrm{ml}$ for a-c respectively. $\mathrm{FIB}$ cross section of particles are shown below. Scale bar for all images except $d$ is $1 \mu \mathrm{m}, d=0.5 \mu \mathrm{m}$. Droplet size was 9.7 $\mu m$

Figure 5a shows the particles obtained using the Pd salt. The particles appear spherical, possess a much more irregular surface than figure s1. FIB liftout cross section was again prepared, and the image shown in figure $5 \mathrm{~d}$. Interestingly within the particles there appears both high and low contrast spots. Being an image formed by collecting back scattered electrons, the brighter spots are corresponding to higher atomic number, whilst the darker ones are associated with lower atomic number material, such as voids. The concentration, and size of the droplets were kept constant and the concentration of the $\mathrm{Fe}_{3} \mathrm{O}_{4}$ varied from 3.3 to 1 and $0.3 \mathrm{mg} / \mathrm{mL}$ for figure $5 \mathrm{ad}$, be, and cf respectively. As the concentration of the $\mathrm{Fe}_{3} \mathrm{O}_{4}$ is decreased the particles become less spherical, appearing wrinkled in the lowest concentration. This in contrast to the smooth spherical particles observed in the absence of the $\mathrm{Pd}$ reaction under the same conditions, figure s1. Elemental analysis on the FIB cross sections for the $\mathrm{Fe}_{3} \mathrm{O}_{4}$ concentrations at 1 and $0.3 \mathrm{mg} / \mathrm{ml}$ are shown in figure 6. 


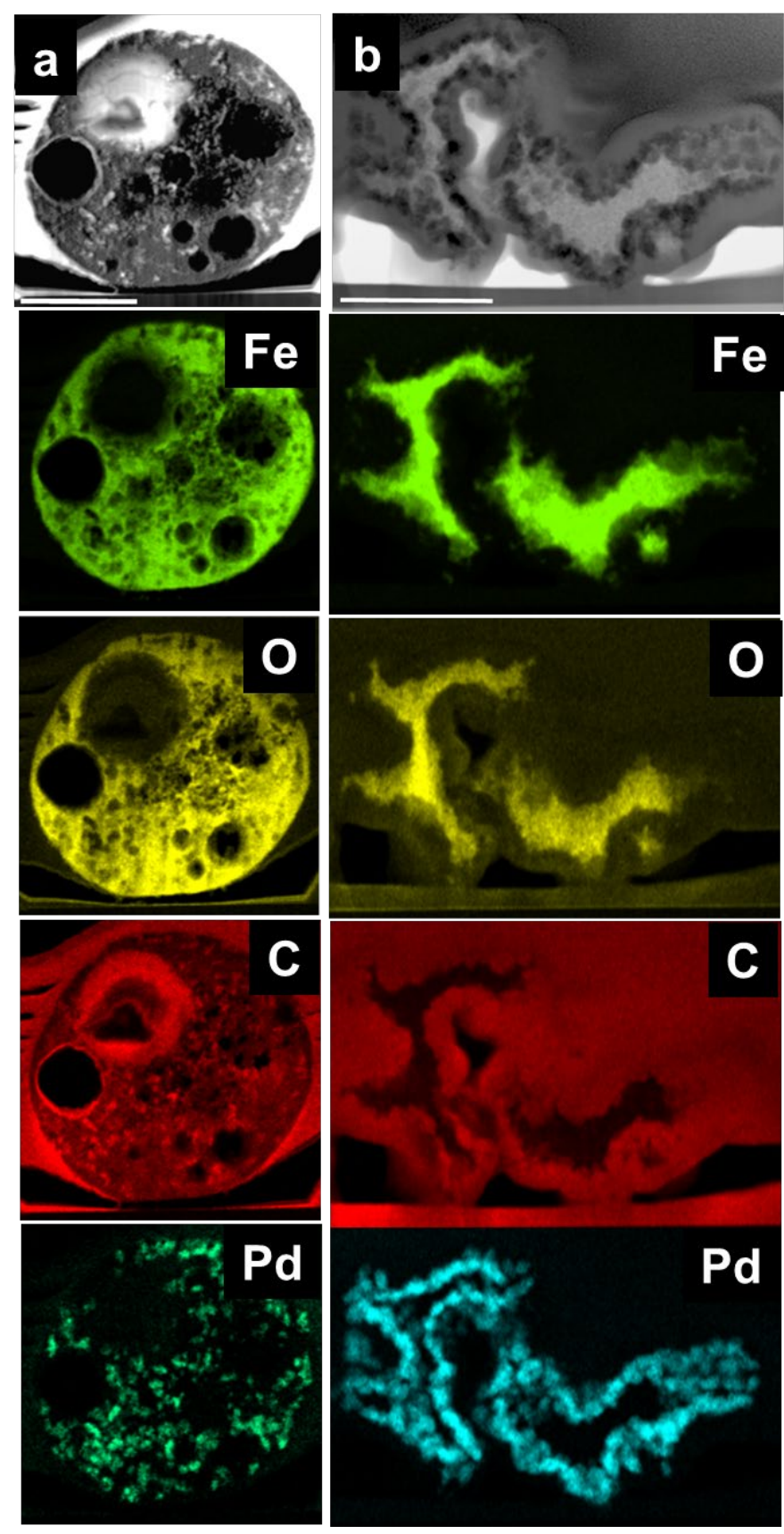

Figure 6.Pd@ $\mathrm{Fe}_{3} \mathrm{O}_{4}$ particle synthesis.DmFc and $\mathrm{K}_{2} \mathrm{PdCl}_{4}$ was $5 \mathrm{mM}$. The concentration of $\mathrm{Fe}_{3} \mathrm{O}_{4}$ was varied from 1 and $0.3 \mathrm{mg} / \mathrm{ml}$ for a $b$ respectively. FIB cross section of particles are shown below. Scale bar for all images except a is $1 \mu \mathrm{m}, b=0.5 \mu \mathrm{m}$. Droplet size was $11 \mu \mathrm{m}$

The darker spots within the particles appear to be voids as evident by the elemental maps, and may have been residual hexane/ water that evaporated during the preparation. Interesting that at the lower concentrations of $\mathrm{Fe}_{3} \mathrm{O}_{4}$ no darker spots were observed, and that the darker spots within figure $6 \mathrm{a}$ has a higher concentration of carbon around the edges. This may have been residual $\mathrm{DmFc}^{+}$that was trapped within the particle. In the absence of any metal deposition the inside of the $\mathrm{Fe}_{3} \mathrm{O}_{4}$ appear uniform, figure s1d. As a control we created carried out the droplet reaction between $\mathrm{DmFc}_{(\text {hex })}$ and hexacyanoferrate $(\mathrm{aq)}$. The resultant particles are shown in figure 
s2. The particles did not appear to be spherical, and often broke apart and fragmented on the SEM imaging stub. The porosity within the particle seems to be related to the presence of the DmFc, and in the absence of any metal NP to help stabilise the droplet the resultant micro particles are fragile. The relationship between the speed of the metal particle formation, the removal of the $\mathrm{Dmfc}^{+}$from the hexane, the speed at which the solvent is removed and the final shape and structure of the core@shell material is clearly complex. Future work to model and understand this mechanism will be needed if it is to be utilised as a common method for the assembly of materials.

Conclusions.

Here we present a simple methodology to synthesise and assemble nanoparticles at and within emulsions droplets. Each droplet acted as microreactor for a reaction between decamethylferrocene $(\mathrm{DmFc})$ within the hexane and metal salts $\left(\mathrm{Ag}^{+} / \mathrm{Pd}^{2+}\right)$ in the aqueous phase. The reaction created metal nanoparticles that were retained at the interface of the droplet for the Ag, however the PdNP's seed to had a weak tertiary structure and did not retain the size of the droplet reactor. The newly formed Pd or AgNP were then attempted to be assembled onto a magnetic core. It was shown that by changing the reaction rate and ratio between palladium and iron oxide a continuous coating of palladium onto iron oxide microspheres can be created. The same reaction with silver, was unsuccessful and resulted in the silver particles being shed into solution, or incorporated within the iron oxide micro particle. This approach could be easily adapted the assembly of other materials but a deeper understanding of the relationship between the speed of the metal particle formation, the removal of the $\mathrm{Dmfc}^{+}$from the hexane, the speed at which the solvent is removed and the final shape and structure of the core@shell material is needed. Our vision for future work is to control the reaction of any metal to form a continuous shell.

Conflicts of interest.

The authors declare no conflict of interest. 


\section{References}

(1) Bardhan, R.; Lal, S.; Joshi, A.; Halas, N. J. Theranostic Nanoshells: From Probe Design to Imaging and Treatment of Cancer. Acc. Chem. Res. 2011, 44 (10), 936-946.

(2) Muehlethaler, C.; Leona, M.; Lombardi, J. R. Review of Surface Enhanced Raman Scattering Applications in Forensic Science. Anal. Chem. 2016, 88 (1), 152-169.

(3) Edel, J. B.; Kornyshev, A. A.; Kucernak, A. R.; Urbakh, M. Fundamentals and Applications of Self-Assembled Plasmonic Nanoparticles at Interfaces. Chem. Soc. Rev. 2016, 45 (6), 1581-1596.

(4) Yuet, K. P.; Hwang, D. K.; Haghgooie, R.; Doyle, P. S. Multifunctional Superparamagnetic Janus Particles. Langmuir 2009, 26 (6), 4281-4287.

(5) Capel, A. J.; Edmondson, S.; Christie, S. D. R.; Goodridge, R. D.; Bibb, R. J.; Thurstans, M. Design and Additive Manufacture for Flow Chemistry. Lab Chip 2013, 13 (23), 4583-4590.

(6) Scanlon, M. D.; Peljo, P.; Mendez, M. A.; Smirnov, E.; Girault, H. H. Charging and Discharging at the Nanoscale: Fermi Level Equilibration of Metallic Nanoparticles. Chem. Sci. 2015, 6 (5), 2705-2720.

(7) Smirnov, E.; Scanlon, M. D.; Momotenko, D.; Vrubel, H.; Méndez, M. A.; Brevet, P.F.; Girault, H. H. Gold Metal Liquid-Like Droplets. ACS Nano 2014, 8 (9), 94719481.

(8) Fang, P.-P.; Chen, S.; Deng, H.; Scanlon, M. D.; Gumy, F.; Lee, H. J.; Momotenko, D.; Amstutz, V.; Cortés-Salazar, F.; Pereira, C. M.; et al. Conductive Gold Nanoparticle Mirrors at Liquid/Liquid Interfaces. ACS Nano 2013, 7 (10), 9241-9248.

(9) Zhang, Y.; Nishi, N.; Sakka, T. Template-Free and Spontaneous Formation of Vertically Aligned Pd Nanofiber Arrays at the Liquid-Liquid Interface between Redox-Active Ionic Liquid and Water. ACS Appl. Mater. Interfaces 2019, 11 (26), 23731-23740.

(10) Zhang, Y.; Nishi, N.; Sakka, T. One-Step Fabrication of Au@Pd Core-Shell Bimetallic Nanofibers at the Interface between Water and Redox-Active Ionic Liquid.

Electrochim. Acta 2019, 325, 134919.

(11) Takagi, S.; Nishi, N.; Sakka, T. Ionic Liquid-in-Water Emulsion-Templated Synthesis of Gold Nanoshells at the Liquid-Liquid Interface between Water and Primary Ammonium-Based Ionic Liquids. Chem. Lett. 2019, 48 (6), 589-592.

(12) Nishi, N.; Yajima, I.; Amano, K.; Sakka, T. Janus-Type Gold/Polythiophene Composites Formed via Redox Reaction at the Ionic Liquid|Water Interface. Langmuir 2018, 34 (7), 2441-2447.

(13) Rao, C. N. R.; Kalyanikutty, K. P. The Liquid-Liquid Interface as a Medium To Generate Nanocrystalline Films of Inorganic Materials. Acc. Chem. Res. 2008, 41 (4), 489-499.

(14) Edel, J. B.; Kornyshev, A. A.; Kucernak, A. R.; Urbakh, M. Fundamentals and Applications of Self-Assembled Plasmonic Nanoparticles at Interfaces. Chem. Soc. Rev. 2016, 45 (6), 1581-1596.

(15) Ramsden, W. Separation of Solids in the Surface-Layers of Solutions and "Suspensions" (Observations on Surface-Membranes, Bubbles, Emulsions, and Mechanical Coagulation). -- Preliminary Account. Proc. R. Soc. London 1903, 72 (477-486), 156-164.

(16) Pickering, S. U. CXCVI.-Emulsions. J. Chem. Soc. Trans. 1907, 91 (0), 2001-2021.

(17) Wu, T.; Wang, H.; Jing, B.; Liu, F.; Burns, P. C.; Na, C. Multi-Body Coalescence in Pickering Emulsions. Nat. Commun. 2015, 6.

(18) Fouilloux, S.; Malloggi, F.; Daillant, J.; Thill, A. Aging Mechanism in Model 
Pickering Emulsion. Soft Matter 2016, 12 (3), 900-904.

(19) Sihler, S.; Schrade, A.; Cao, Z.; Ziener, U. Inverse Pickering Emulsions with Droplet Sizes below 500 Nm. Langmuir 2015, 31 (38), 10392-10401.

(20) Nie, Z.; Park, J. Il; Li, W.; Bon, S. A. F.; Kumacheva, E. An "Inside-Out" Microfluidic Approach to Monodisperse Emulsions Stabilized by Solid Particles. J. Am. Chem. Soc. 2008, 130 (49), 16508-16509.

(21) Sachdev, S.; Maugi, R.; Kirk, C.; Zhou, Z.; Christie, S. D. R.; Platt, M. Synthesis and Assembly of Gold and Iron Oxide Particles Within an Emulsion Droplet; Facile Production of Core@Shell Particles. Colloid Interface Sci. Commun. 2017, 16, 14-18.

(22) Sachdev, S.; Maugi, R.; Woolley, J.; Kirk, C.; Zhou, Z.; Christie, S. D. R.; Platt, M. Synthesis of Gold Nanoparticles Using the Interface of an Emulsion Droplet. Langmuir 2017, 33 (22), 5464-5472.

(23) Platt, M.; Dryfe, R. A. W.; Roberts, E. P. L. Controlled Deposition of Nanoparticles at the Liquid-Liquid Interface. Chem. Commun. 2002, No. 20, 2324-2325.

(24) Platt, M.; Dryfe, R. A. W.; Roberts, E. P. L. Electrodeposition of Palladium Nanoparticles at the Liquid-Liquid Interface Using Porous Alumina Templates. Electrochim. Acta 2003, 48 (20-22).

(25) Sherburn, A.; Platt, M.; Arrigan, D. W. M.; Boag, N. M.; Dryfe, R. A. W. Selective Silver Ion Transfer Voltammetry at the Polarised Liquid|liquid Interface. Analyst 2003, $128(9), 1187-1192$. 
TOC only

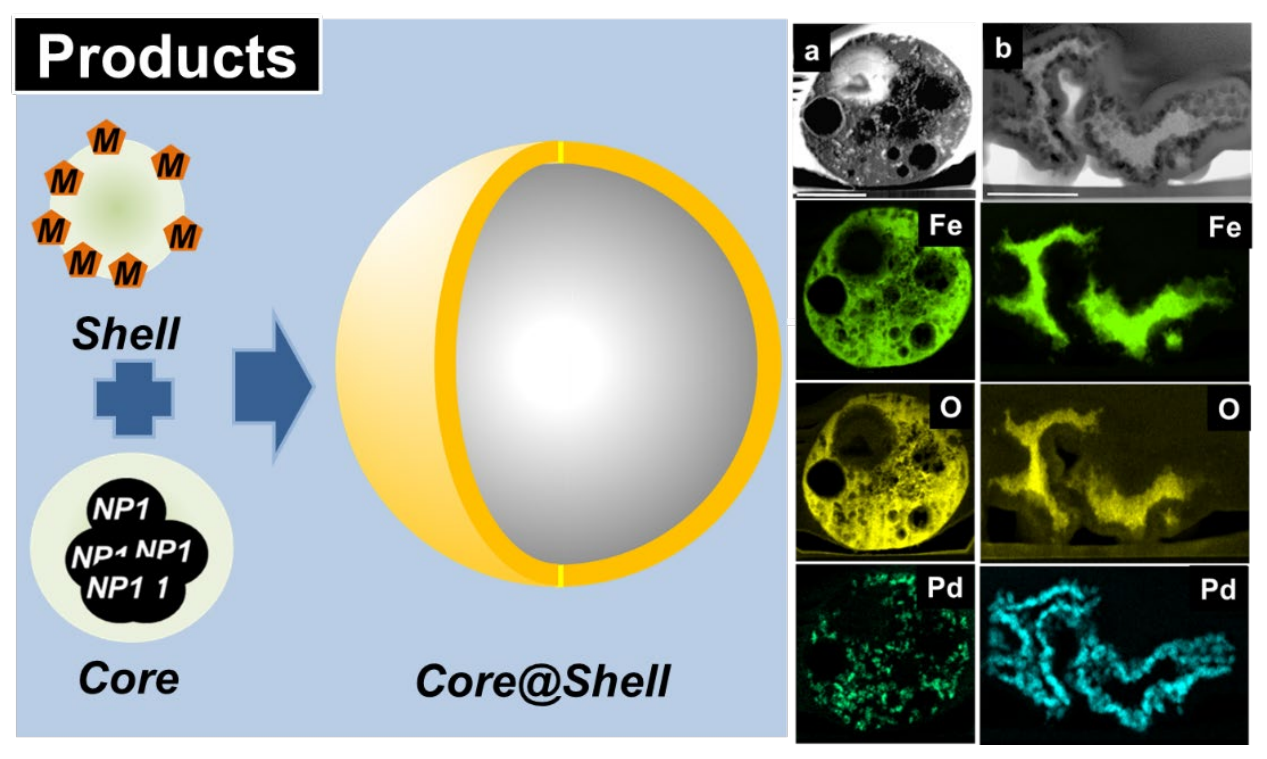

